\title{
Clinical outcomes of surgical resection for recurrent lesion after curative esophagectomy for esophageal squamous cell carcinoma: a nationwide, large-scale retrospective study
}

\author{
Kensuke Kudou ${ }^{1,2} \cdot$ Hiroshi Saeki $^{1,3} \cdot$ Yuichiro Nakashima $^{1} \cdot$ Yasue Kimura $^{1} \cdot$ Eiji Oki $^{1} \cdot$ Masaki Mori $^{1,4}$. \\ Mototsugu Shimokawa ${ }^{5,6} \cdot$ Yoshihiro Kakeji $^{7} \cdot$ Yasushi Toh $^{8} \cdot$ Yuichiro Doki $^{9} \cdot$ Hisahiro Matsubara ${ }^{10}$
}

Received: 25 May 2021 / Accepted: 6 September 2021 / Published online: 12 September 2021

(C) The Author(s) 2021

\begin{abstract}
Background Several studies have reported the efficacy of resection for recurrent lesions. However, they involved a limited number of subjects. This study aimed to identify a subset of patients who benefit from surgical resection of recurrent lesions after curative esophagectomy for esophageal squamous cell carcinoma.

Methods Clinicopathological features of 186 patients with esophageal squamous cell carcinoma who underwent surgical treatment for postoperative recurrent lesions at 37 accredited institutions of the Japanese Esophageal Society were evaluated. Results The most common recurrence site was the lymph node (106 cases; 58.6\%), followed by the lung ( 40 cases; $22.1 \%)$. Univariate analyses revealed that $\mathrm{pN} 0-1$ at esophagectomy $(P=0.0348)$, recurrence-free interval of $\geq 550$ days $(P=0.0306)$, R0 resection $(P<0.0001)$, and absence of severe complications after resection for recurrent lesions (ClavienDindo grade $<$ IIIa) $(P=0.0472)$ were associated with better overall survival after surgical resection. According to multivariate analyses, $\mathrm{pN} 0-1(P=0.0146)$, lung metastasis $(P=0.0274)$, recurrence-free interval after curative esophagectomy of $\geq 550$ days $(P=0.0266)$, R0 resection $(P=0.0009)$, and absence of severe complications after resection for recurrent lesions (Clavien-Dindo grade $<$ IIIa) $(P=0.0420)$ were independent predictive factors for better overall survival.

Conclusions Surgical resection of recurrent esophageal squamous cell carcinoma lesions is a useful option, especially for cases involving lower $\mathrm{pN}$ stage, lung metastasis, long recurrence-free intervals after esophagectomy, and technically resectable lesions. Surgical risks should be minimized as much as possible.
\end{abstract}

Keywords Esophageal squamous cell carcinoma $\cdot$ Esophagectomy $\cdot$ Recurrence

Hiroshi Saeki

h-saeki@gunma-u.ac.jp

1 Department of Surgery and Science, Graduate School of Medical Sciences, Kyushu University, Fukuoka, Japan

2 Department of Gastroenterological Surgery, Clinical Research Institute Cancer Research Division, National Hospital Organization Kyushu Medical Center, Fukuoka, Japan

3 Department of General Surgical Science, Gastroenterological Surgery, Graduate School of Medicine, Gunma University, 3-39-22 Showa-machi, Maebashi, Gunma 371-8511, Japan

4 School of Medicine, Tokai University, Tokyo, Japan

5 Department of Biostatistics, Yamaguchi University Graduate School of Medicine, Yamaguchi, Japan

6 Clinical Research Institute, National Hospital Organization Kyushu Cancer Center, Fukuoka, Japan

7 Division of Gastrointestinal Surgery, Department of Surgery, Graduate School of Medicine, Kobe University, Kobe, Japan

8 Department of Gastroenterological Surgery, National Hospital Organization Kyushu Cancer Center, Fukuoka, Japan

9 Department of Gastroenterological Surgery, Graduate School of Medicine, Osaka University, Suita, Osaka, Japan

10 Department of Frontier Surgery, Graduate School of Medicine, Chiba University, Chiba, Japan 


\section{Introduction}

Recurrence after radical surgery (R0 resection) for esophageal cancer occurs in $28-47 \%$ of patients in Japan [1], and several studies performed in Western countries have reported recurrence rates of $>50 \%$ [2-4]. The median time to recurrence was 10-12 months, and the median survival time was 7-18 months $[4,5]$. A previous study reported that $90 \%$ of recurrent esophageal cancer cases occurred within 38 months after surgery, and that half of those cases recurred within 12 months [4]. Long-term survival and complete cure, especially for the subset of patients who underwent successful surgical resection for lymph node (LN) or lung recurrence, have been reported; therefore, aggressive treatment for recurrent diseases could be considered for some patients [4-11].

The significance of local treatment for recurrent esophageal cancer has not yet been ascertained. When recurrence occurs in localized or resectable regions after radical esophagectomy, surgical resection, radiotherapy, and chemoradiotherapy are considered effective treatments [12]. Recurrence is frequently observed in the LNs, lungs, liver, and bone [13]. Other studies reported that anastomotic LNs, supraclavicular LNs, and mediastinal LNs were the most common local recurrence sites $[4,14,15]$. Several studies have demonstrated the efficacy of lymphadenectomy for localized LN recurrence, such as cervical LNs, but these studies were performed at a single center or involved only a limited number of cases [6-10]. Regarding resection for local recurrence and recurrent lesions of other organs, such as the lung, liver, brain, parotid gland, and pancreas, only reports based on a small number of cases are available; therefore, its efficacy is still undetermined [11, 16-18]. $\mathrm{Su}$ et al. reported that the time of recurrence after curative esophagectomy ( $<12$ months), pattern of recurrence (local-regional recurrence defined as anastomotic recurrence and/or occurring in the mediastinum, upper abdomen, or cervical area), and treatment of recurrence (radiotherapy, surgery, or chemotherapy) were independent favorable prognostic factors for patients with recurrence after complete resection of esophageal carcinoma [13]. Of note, during this study, treatments for recurrent lesions included a mixture of modalities such as radiotherapy, chemotherapy, chemoradiotherapy, and radiofrequency ablation, and the proportion of patients who underwent surgical resection was approximately 5\% [13]. Therefore, the clinical significance of surgical resection for recurrence in patients with esophageal carcinoma remains controversial. It is important to investigate the indications for surgical resection and the efficacy of surgical resection for recurrence, and to identify subgroups in which a favorable long-term prognosis can be expected after resecting the recurrent lesions.
We conducted a nationwide survey of patients with recurrent esophageal squamous cell carcinoma (ESCC) treated at institutions accredited by the Japan Esophageal Society. We focused on ESCC, which accounts for 90\% of the histological types in Japan, to avoid confusion with different types of carcinomas. This study aimed to clarify the current state of resection of recurrent lesions and to identify a subset of patients who can be expected to have a favorable long-term prognosis. To our knowledge, this is the largest study of surgery for recurrent ESCC to date, and we believe that the results will be of great significance to practical treatment strategies.

\section{Methods}

\section{Study design and patients}

This study was approved as a research project by the Japanese Esophageal Society in 2017. We conducted a questionnaire-based, retrospective clinical review of patients with recurrent ESCC who underwent surgical treatment for recurrent lesions after curative esophagectomy at institutes accredited by the Japan Esophageal Society between January 2009 and December 2013. Of the 105 facilities, we received responses from 58; 21 of these 58 facilities had no applicable cases. Finally, the data from 37 facilities were analyzed during the present study. The questionnaire was prepared at Kyushu University, approved by the ethics review committee, and then sent to the participating facilities of the Japan Esophageal Society. Characteristics such as sex, age, tumor location, clinical stage, pathological stage, comorbidities, surgical procedure, pathological effect of neoadjuvant therapy, postoperative complications, details of perioperative therapy, number of recurrent lesions, recurrence site, residual tumor, recurrence-free interval, and longterm prognosis were investigated using this questionnaire. Cancer staging was based on the TNM classification. The clinical and pathological data of 186 patients with histologically diagnosed ESCC who underwent surgical treatment for postoperative recurrent lesions were collected from the participating institutions. Patients with adenocarcinoma or other tumors were excluded from the study. Permission to perform this retrospective cohort study was provided by the Institutional Review Board of Kyushu University (29-325) and the other 36 institutions.

\section{Statistical analyses}

Survival curves were plotted according to the Kaplan-Meier method, and differences were analyzed using the log-rank test and a Cox proportional hazard model. Overall survival (OS) during this study was measured from the date of 
surgical resection for recurrent lesions. Univariate and multivariate analyses were performed using a Cox proportional hazards model to identify independent prognostic factors. All $P$ values were two-sided, and $P<0.05$ was considered statistically significant. A receiver-operating characteristic curve analysis was used to identify optimal cut-off values. All analyses were performed using JMP PRO 11 software (SAS Institute Inc., Cary, NC; https://www.jmp.com/ja_jp/ home.html).

\section{Results}

\section{Patient characteristics at the time of initial esophagectomy for primary ESCC}

The clinicopathological characteristics of 186 ESCC patients (159 [85.5\%] men and 27 [14.5\%] women) are summarized in Table 1. The median patient age at the time of primary esophagectomy was 63.3 years (range, $40-83$ years). The middle thorax was the most frequent $(48.4 \%)$ tumor location, followed by the lower thorax (25.3\%), upper thorax $(16.7 \%)$, cervix (4.8\%), and abdomen (4.8\%). Regarding the surgical procedure for the primary lesion, 167 (89.8\%) patients underwent subtotal esophagectomy and reconstruction. Postoperative complications occurred in 93 (50.0\%) patients after surgery for the primary lesion. The degree of postoperative complications was categorized according to the Clavien-Dindo classification [19, 20]. Severe complications (Clavien-Dindo grade $\geq$ IIIa) occurred in 19.9\%. Major complications included recurrent nerve paralysis (13.4\%), respiratory complications (12.9\%), and anastomotic leakage $(10.8 \%)$. Details of neoadjuvant and adjuvant therapies associated with esophagectomy are summarized in Online Resource 1.

\section{Clinical features at the time of postoperative recurrence}

The clinical features related to surgery for recurrent lesions are summarized in Table 2. The median interval from the date of prior esophagectomy to the date of recurrence was 351 days (range, 57-3556 days). Regarding the number of recurrent lesions targeted for resection, 148 cases $(79.6 \%)$ involved solitary lesions in a single organ, $24(12.9 \%)$ involved multiple lesions in a single organ, and $14(7.5 \%)$ involved multiple lesions in multiple organs. Among the 186 patients, 157 (84.4\%) underwent complete resection (R0 resection), 12 (6.5\%) had microscopic residual disease (R1 resection), and 17 (9.1\%) had macroscopic residual disease ( $\mathrm{R} 2$ resection). The incidence of postoperative complications for recurrent lesions was lower than that after surgery for primary esophagectomy
Table 1 Clinicopathological characteristics of patients at initial esophagectomy

\begin{tabular}{|c|c|c|}
\hline \multirow[t]{2}{*}{ Characteristic } & \multicolumn{2}{|c|}{ No. of patients } \\
\hline & $n$ & $(\%)$ \\
\hline \multicolumn{3}{|l|}{ Sex } \\
\hline Male & 159 & $(85.5)$ \\
\hline Female & 27 & $(14.5)$ \\
\hline \multicolumn{3}{|l|}{ Age } \\
\hline 63.3 & & $(40-83)$ \\
\hline \multicolumn{3}{|l|}{ Tumor location } \\
\hline Cervix & 9 & $(4.8)$ \\
\hline Upper thorax & 31 & $(16.7)$ \\
\hline Middle thorax & 90 & $(48.4)$ \\
\hline Lower thorax & 47 & $(25.3)$ \\
\hline Abdomen & 9 & $(4.8)$ \\
\hline \multicolumn{3}{|l|}{ cStage } \\
\hline I & 23 & (12.4) \\
\hline II & 61 & $(32.8)$ \\
\hline III & 88 & $(47.3)$ \\
\hline IVa & 14 & $(7.5)$ \\
\hline \multicolumn{3}{|l|}{$c T$} \\
\hline $\mathrm{T} 1$ & 43 & $(23.1)$ \\
\hline $\mathrm{T} 2$ & 38 & $(20.4)$ \\
\hline $\mathrm{T} 3$ & 92 & $(49.5)$ \\
\hline $\mathrm{T} 4$ & 13 & $(7.0)$ \\
\hline \multicolumn{3}{|l|}{$c N$} \\
\hline No & 53 & $(28.5)$ \\
\hline N1 & 57 & $(30.6)$ \\
\hline $\mathrm{N} 2$ & 47 & $(25.3)$ \\
\hline N3 & 19 & $(10.2)$ \\
\hline N4 & 10 & $(5.4)$ \\
\hline \multicolumn{3}{|l|}{ Comorbidities and previous history } \\
\hline No & 114 & $(61.3)$ \\
\hline Cardiovascular disease & 17 & $(9.1)$ \\
\hline Hypertension & 17 & $(9.1)$ \\
\hline Respiratory disease & 13 & $(7.0)$ \\
\hline Malignant tumor & 11 & $(5.9)$ \\
\hline Diabetes & 10 & (5.4) \\
\hline Liver disease & 7 & (3.8) \\
\hline Cerebrovascular disease & 6 & $(3.2)$ \\
\hline Others & 9 & $(4.8)$ \\
\hline \multicolumn{3}{|l|}{ Surgical procedure } \\
\hline Subtotal esophagectomy & 167 & $(89.8)$ \\
\hline Laryngopharyngoesophagectomy & 7 & $(3.8)$ \\
\hline Others & 12 & $(6.5)$ \\
\hline \multicolumn{3}{|l|}{ All postoperative complications } \\
\hline No & 93 & $(50.0)$ \\
\hline Yes & 93 & $(50.0)$ \\
\hline \multicolumn{3}{|c|}{ Severe complications $(C D$ grade $\geq I I I a)$} \\
\hline No & 149 & $(80.1)$ \\
\hline Yes & 37 & $(19.9)$ \\
\hline
\end{tabular}


Table 1 (continued)

\begin{tabular}{|c|c|c|}
\hline \multirow[t]{2}{*}{ Characteristic } & \multicolumn{2}{|c|}{ No. of patients } \\
\hline & $n$ & $(\%)$ \\
\hline \multicolumn{3}{|l|}{ Details of complications } \\
\hline No & 93 & $(50.0)$ \\
\hline Recurrent nerve paralysis & 25 & $(13.4)$ \\
\hline Respiratory complication & 24 & $(12.9)$ \\
\hline Anastomotic leakage & 20 & $(10.8)$ \\
\hline Surgical site infection & 15 & $(8.1)$ \\
\hline Cardiovascular complication & 12 & $(6.5)$ \\
\hline Chylothorax & 9 & $(4.8)$ \\
\hline Bleeding & 4 & $(2.2)$ \\
\hline Others & 16 & $(8.6)$ \\
\hline \multicolumn{3}{|l|}{ pStage } \\
\hline 0 & 12 & $(6.5)$ \\
\hline I & 26 & $(14.0)$ \\
\hline II & 55 & $(29.6)$ \\
\hline III & 81 & $(43.5)$ \\
\hline IVa & 12 & $(6.5)$ \\
\hline \multicolumn{3}{|l|}{$p T$} \\
\hline T0 & 13 & $(7.0)$ \\
\hline $\mathrm{T} 1$ & 58 & $(31.2)$ \\
\hline $\mathrm{T} 2$ & 22 & $(11.8)$ \\
\hline $\mathrm{T} 3$ & 84 & $(45.2)$ \\
\hline $\mathrm{T} 4$ & 9 & $(4.8)$ \\
\hline \multicolumn{3}{|l|}{$p N$} \\
\hline N0 & 64 & $(34.4)$ \\
\hline N1 & 45 & $(24.2)$ \\
\hline $\mathrm{N} 2$ & 50 & $(26.9)$ \\
\hline N3 & 21 & $(11.3)$ \\
\hline N4 & 6 & $(3.2)$ \\
\hline \multicolumn{3}{|c|}{ Pathological effect of neoadjuvant therapy $(n=111)$} \\
\hline Grade 0 & 13 & $(7.0)$ \\
\hline Grade 1a & 51 & $(27.4)$ \\
\hline Grade $1 b$ & 12 & $(6.5)$ \\
\hline Grade 2 & 16 & $(8.6)$ \\
\hline Grade 3 & 12 & $(6.5)$ \\
\hline Unknown & 7 & (3.8) \\
\hline
\end{tabular}

Data are presented as $n(\%)$, with the exception of age, which is presented as mean (range)

$C D$ Clavien-Dindo classification

(all grades: $10.2 \%$ vs. $50.0 \%$; severe [Clavien-Dindo grade $\geq$ IIIa]: $3.8 \%$ vs. $19.9 \%$ ). Severe complications after surgery for recurrent lesions included respiratory complications, bleeding, recurrent nerve paralysis, pyothorax, and necrosis of the reconstructed intestine. Recurrence most frequently occurred in the LN (108 cases; $58.1 \%$ ), followed by the lung ( 42 cases; $22.6 \%$ ), brain ( 7 cases; $3.8 \%$ ), skin ( 6 cases; $3.2 \%$ ), and liver and adrenal glands ( 5 cases; $2.7 \%$ ) (Fig. 1b).
Table 2 Clinical features related to surgery for recurrent lesion

\begin{tabular}{|c|c|c|}
\hline \multirow[t]{2}{*}{ Characteristic } & \multicolumn{2}{|c|}{ No. of patients } \\
\hline & $n$ & $(\%)$ \\
\hline \multicolumn{3}{|l|}{ Number of recurrent lesions } \\
\hline Solitary & 148 & (79.6) \\
\hline Multiple & 38 & $(20.4)$ \\
\hline \multicolumn{3}{|c|}{ Number of organs with recurrence } \\
\hline Single & 172 & $(92.5)$ \\
\hline Multiple & 14 & $(7.5)$ \\
\hline \multicolumn{3}{|l|}{ Residual tumor } \\
\hline R0 & 157 & $(84.4)$ \\
\hline $\mathrm{R} 1$ & 12 & $(6.5)$ \\
\hline $\mathrm{R} 2$ & 17 & $(9.1)$ \\
\hline \multicolumn{3}{|c|}{ All postoperative complications } \\
\hline No & 167 & $(89.8)$ \\
\hline Yes & 19 & $(10.2)$ \\
\hline \multicolumn{3}{|c|}{ Severe complications $(C D$ grade $\geq I I I a)$} \\
\hline No & 179 & $(96.2)$ \\
\hline Yes & 7 & (3.8) \\
\hline \multicolumn{3}{|l|}{ Details of complications } \\
\hline No & 167 & $(89.8)$ \\
\hline Recurrent nerve paralysis & 5 & $(2.7)$ \\
\hline Respiratory complication & 3 & (1.6) \\
\hline Bleeding & 3 & (1.6) \\
\hline Surgical site infection & 3 & (1.6) \\
\hline Others & 5 & $(2.7)$ \\
\hline
\end{tabular}

$C D$ Clavien-Dindo classification

The details of therapy before and after surgical resection for recurrent lesions were also investigated. Twenty-five percent of patients underwent chemotherapy and/or radiotherapy before surgical resection for recurrent lesions. Therapy after surgery for recurrent lesions was performed for $45 \%$ of patients. Details about chemotherapy regimens and radiation exposure doses are shown in Online Resource 2.

\section{Survival based on recurrence location}

Among the 186 ESCC patients, 5 were excluded from the prognosis analyses during this study because of the lack of available data regarding survival time. Therefore, for 181 patients, the median survival time after surgery for recurrent lesions was 1030 days (range, 64-2748 days) and the 5-year OS rate was $39.8 \%$ (Fig. 1a). Patients were divided into three groups according to the recurrence location (LN, lung, or other organs), and the Kaplan-Meier method was used to compare the OS after surgery for recurrent lesions. The 5-year OS rates were better for patients who underwent surgical resection of the $\mathrm{LN}$ or lung than for those who underwent surgical resection of other organs (LN, 39.5\%; lung, 54.5\%; other organs, $27.9 \% ; P=0.0102$ ) (Fig. 1c). 
Between-group comparisons indicated that the prognosis for patients whose target lesions were in the LN or lung was significantly better than that for patients with target lesions in other organs (hazard ratio [HR]: LN vs. others, 0.555, $P=0.0159$; HR: lung vs. others, $0.440, P=0.0065$; HR: lung vs. LN, $0.792, P=0.3771)$.

Subgroup analyses were performed. The Kaplan-Meier method was performed for subjects with LN metastases by categorizing them into three groups according to the recurrent lesion location (cervix, thorax, and abdomen). Patients with thoracic LN metastases had a relatively poor prognosis compared to patients with recurrence in the other two locations ( $P=0.0843$ ) (Fig. 1d). Comparisons of each group indicated that the prognosis for the thorax group was significantly poorer than that for the cervix group (HR: thorax vs. cervix, 1.865, $P=0.0354$; HR: thorax vs. abdomen, 1.661, $P=0.2132$; HR: abdomen vs. cervix, $1.123, P=0.7736$ ).

\section{Survival after recurrent lesion resection}

The Kaplan-Meier method was performed by categorizing the patients into two groups according to the $\mathrm{pN}$ stage at primary esophagectomy ( $\mathrm{pN} 0-1$ vs. pN2-4), the number of resected recurrent organs (single organ vs. multiple organs), or whether complete resection for recurrent lesions was achieved (R0 surgery vs. R1 or R2 surgery). The results revealed that $\mathrm{pN} 0$ and $\mathrm{pN} 1(\mathrm{HR}, 0.659 ; P=0.0326)$ and $\mathrm{R} 0$ surgery (HR, 0.299; $P<0.0001)$ were significantly correlated with better prognoses (Fig. 2a, c), and that single-organ metastasis (HR, 0.626; $P=0.1389$ ) tended to be correlated with better OS, but not significantly (Fig. 2b).

We analyzed the relationship between the length of the recurrence-free interval after primary esophagectomy and prognosis after recurrence. The cut-off value was identified as 550 days by the receiver-operating characteristic curve analysis. Patients whose recurrence-free interval was $\geq 550$ days had a better prognosis after resection of the recurrent lesion (HR, 0.604; $P=0.0370)$ (Fig. 2d).

\section{Survival according to the development of complications after recurrent lesion resection}

We also analyzed the correlation between the prognosis and the occurrence of complications after surgical resection for recurrent lesions. The OS rate of patients with complications after surgical resection for recurrent lesions tended to be poorer than that of patients without complications $(P=0.0959)$ (Fig. 3a). The OS rate of those with severe complications (Clavien-Dindo grade $\geq$ IIIa) was significantly poorer than that of patients without severe complications $(P=0.0186)$ (Fig. 3b).

\section{Efficacy of therapy before and after surgical resection for recurrent lesions}

To verify the efficacy of perioperative chemotherapy and/ or radiotherapy after recurrence, patients were divided into two groups according to the use of chemotherapy and/or radiotherapy before or after surgical resection for recurrent lesions and their OS rates were compared. The Kaplan-Meier curves showed that perioperative chemotherapy and/or radiotherapy did not improve OS after surgical resection for recurrent lesions (Fig. 4). The clinicopathological characteristics of patients according to treatment with chemotherapy and/or radiotherapy before and after surgical resection for recurrent lesions are summarized in Online Resources 3-6. There were no significant differences in clinical features between the two groups categorized according to the treatment before surgery for recurrent lesions (Online Resources 3 and 4). On the other hand, the rates of $\mathrm{R} 0$ resection $(P=0.0069)$ and single-organ recurrence $(P=0.0095)$ were significantly lower in patients who underwent treatment after surgery for recurrent lesions than in those who did not undergo postoperative treatment (Online Resources 5 and 6). Subgroup analyses were also performed according to the number of recurrent lesions and organs or the location of recurrent lesions, and no significant difference in the OS rates was observed (Online Resources 7-9).

\section{Factors associated with favorable prognoses after recurrent lesion resection}

To identify independent prognostic factors for favorable OS after recurrent lesion resection, univariate and multivariate analyses with a Cox proportional hazards model were performed. If the $P$ value of a factor was $<0.1$ in the univariate analyses, then we included that factor in the multivariate analyses. Univariate analyses revealed that $\mathrm{pN} 0$ and $\mathrm{pN} 1$ at esophagectomy $(P=0.0348)$, recurrence-free interval of $\geq 550$ days $(P=0.0306), \mathrm{R} 0$ resection $(P<0.0001)$, and absence of severe complications after resection for recurrent lesions (Clavien-Dindo grade $<$ IIIa) $(P=0.0472)$ were associated with better OS after surgical resection for recurrent lesions. We also performed univariate analyses using $\mathrm{pN} 0$ as the control. HR was 0.779 for $\mathrm{pN} 0$ vs pN1-4 $(P=0.2392)$ and 0.669 for $\mathrm{pN} 0$ vs $\mathrm{pN} 2-4(P=0.0747)$. In the multivariate analyses, $\mathrm{pN} 0$ and $\mathrm{pN} 1$ (HR, 0.594; $P=0.0146)$, lung metastasis (HR, 0.529; $P=0.0274)$, recurrence-free interval of $\geq 550$ days (HR, $0.584 ; P=0.0266)$, R0 resection (HR, 
(a)

All patients

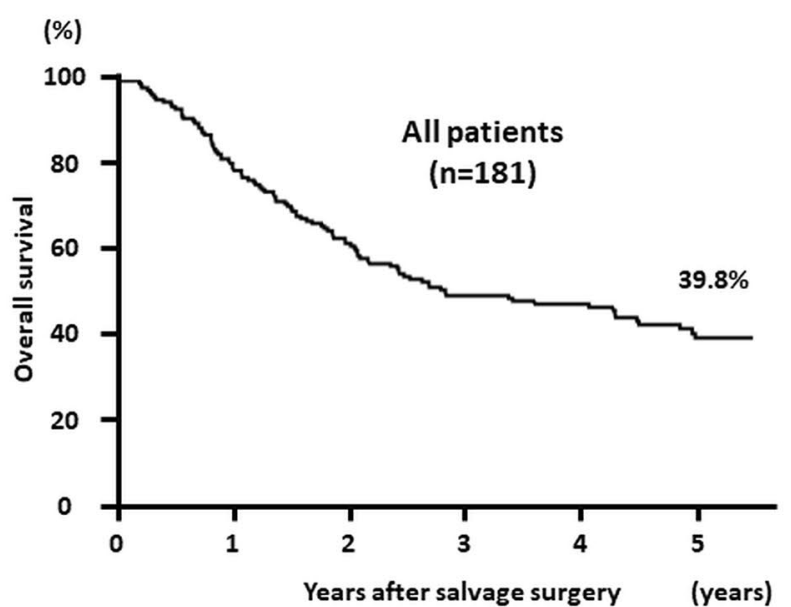

Number at risk

$\begin{array}{lllllll}\text { All patients } & 181 & 142 & 104 & 78 & 64 & 37\end{array}$

(c)

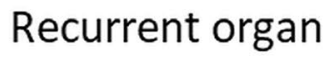

(\%)

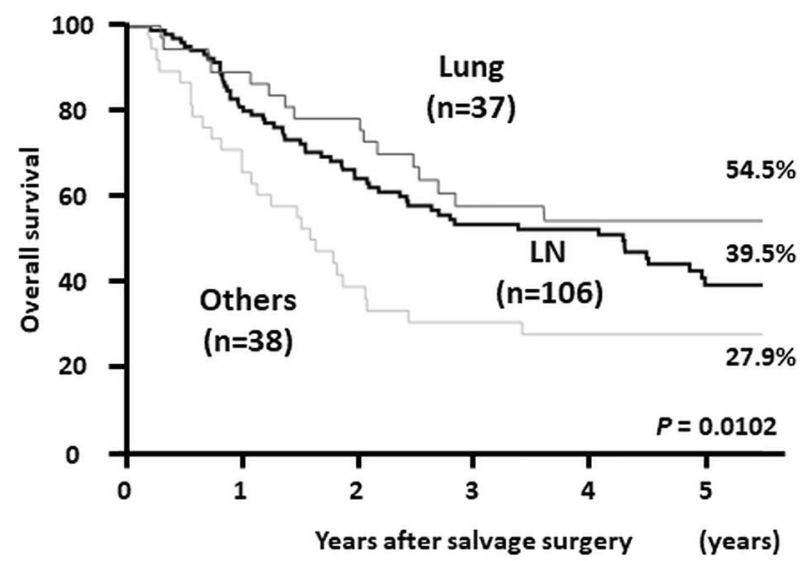

Number at risk

\begin{tabular}{|c|c|c|c|c|}
\hline Lung & 37 & 33 & 28 & 19 \\
\hline LN & 106 & 84 & 62 & 48 \\
\hline \multirow[t]{5}{*}{ Others } & 38 & 25 & 14 & 11 \\
\hline & & & & MST (days) \\
\hline & & & Lung & 2187 \\
\hline & & & LN & 1557 \\
\hline & & & Others & 581.5 \\
\hline
\end{tabular}

\begin{tabular}{|l|c|}
\hline & MST (days) \\
\hline All patients & 1030 \\
\hline
\end{tabular} (b)

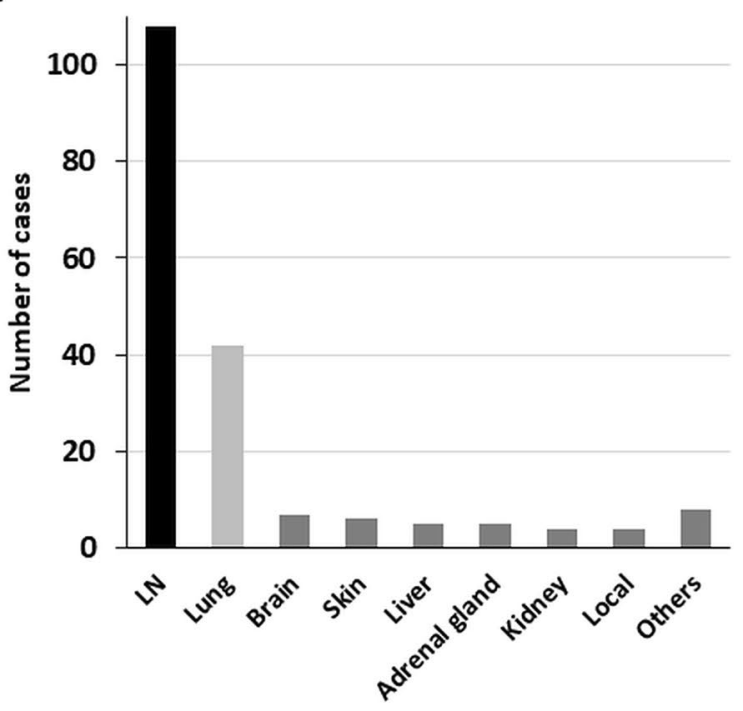

Recurrent organ (d) Location of recurrent LN

(\%)

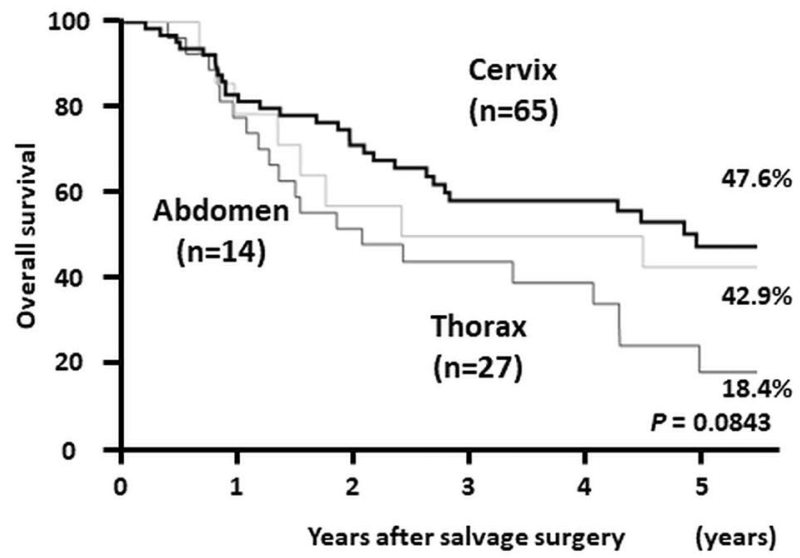

Number at risk

$\begin{array}{ll}\text { Cervix } & 65 \\ \text { Thorax } & 27\end{array}$

Abdomen 14

$\begin{array}{ccccc}52 & 40 & 31 & 26 & 16 \\ 21 & 14 & 10 & 8 & 4 \\ 11 & 8 & 7 & 7 & 5\end{array}$

\begin{tabular}{|c|c|}
\hline & MST (days) \\
\hline Cervix & 1805 \\
\hline Thorax & 752 \\
\hline Abdomen & 1257 \\
\hline
\end{tabular}


४Fig. 1 a Overall survival (OS) after recurrence for 181 patients. The median survival time after surgical resection for recurrent lesions was 1030 days and the 5-year OS rate was $39.8 \%$. b Categorization by organs and number of cases. c Kaplan-Meier curves of OS after surgical resection for recurrent lesions among three groups created based on the organs where recurrence was observed (lymph nodes, lung, and other organs). d Kaplan-Meier curves of OS after surgical resection for recurrent lesions among patients with lymph-node metastases categorized into three groups created according to the location of recurrent lesions (cervix, thorax, and abdomen). $L N$ lymph node, MST median survival time

0.389; $P=0.0009)$, and absence of severe complications after recurrent lesion resection (Clavien-Dindo grade $<$ IIIa) (HR, 0.352; $P=0.0420$ ) were independent predictive factors for better OS for patients who underwent surgical resection of recurrent lesions (Table 3).

\section{R0 resection rate by each site of recurrence}

The results of univariate and multivariate analyses indicated that $\mathrm{R} 0$ resection showed a strong correlation with better OS after surgical resection of recurrent lesions (Table 3). On the basis of this finding, we investigated the $\mathrm{R} 0$ resection rate by each site of recurrence. With regard to LN metastases according to the site of the recurrent lesion (cervix, thorax, and abdomen), the $\mathrm{R} 0$ resection rate was lower for thoracic LN metastases than for cervical and abdominal LN metastases. With regard to other organs, the $\mathrm{R} 0$ resection rate was higher for the lungs (95\%) and liver (100\%) than for other organs (Online Resource 10).

\section{Discussion}

When recurrence occurs in a localized region or regions after radical surgery for esophageal cancer, surgery, chemoradiotherapy, and radiotherapy are considered treatments that can lead to radical cure [12]. Several studies that evaluated the efficacy of surgical resection for recurrent lesions after radical esophagectomy have suggested that resection of the LN or lung metastases yields a survival benefit for properly selected patients, but the benefit of resection for hepatic metastases remains controversial [7, 9, 11, 18]. A few studies indicated that successful resection for metastases of other organs, such as the brain, parotid gland, or pancreas, or for local recurrence may improve prognosis, although these results were based on one or a few cases $[16,17]$. Many studies have highlighted the usefulness of chemoradiotherapy and radiotherapy for localized recurrence after radical surgery [21-24], and they are widely used in actual clinical practice. However, Ma et al. retrospectively compared the long-term outcomes of lymphadenectomy for cervical LN recurrence compared with those of chemoradiotherapy/radiotherapy and reported that patients who underwent lymphadenectomy had a better prognosis than those who received chemoradiotherapy/radiotherapy [10]. Most previous studies (including our study) demonstrated that the 5-year survival rates were 3-13\% for patients who underwent chemoradiotherapy/radiotherapy for recurrent esophageal cancer [10, 21-24], and that their outcomes were poorer than those of patients who underwent surgical resection for recurrent lesions. However, no prospective comparative study has evaluated a suitable treatment strategy for postoperative recurrent ESCC.

Some small cohort studies have suggested that surgical resection might be useful, especially for localized recurrence in the LNs and lung in selected patients. However, the characteristics of ESCC patients who would benefit from recurrent lesion resection have not been ascertained. Therefore, it is necessary to identify the subset of patients who would benefit from surgical resection and identify the indications for surgery. Previous studies have reported that a higher primary $\mathrm{N}$ stage, shorter disease-free survival after primary esophagectomy, and multiple recurrences in cervical LNs were associated with poor prognoses after salvage treatment [25-27]. Moreover, Wang et al. analyzed 66 cases of salvage lymphadenectomy for ESCC recurrence in the cervical LNs and observed that a lower primary $\mathrm{N}$ stage and $\mathrm{R} 0$ resection of recurrent nodes were favorable prognostic factors [9]. During the present study, we investigated the outcomes of surgical resection of various organ metastases in a large cohort. Our results suggest that lower $\mathrm{pN}$ stage, lung metastasis, long recurrence-free interval after esophagectomy, and technically resectable lesions are favorable prognostic factors for OS after resection of recurrent lesions in ESCC patients; these results are consistent with those of previous studies. In particular, R0 resection showed a strong correlation with better OS after surgical resection of recurrent lesions. Accordingly, we compared the $\mathrm{R} 0$ resection rate by each site of recurrence (Online Resource 10) and found a lower rate for thoracic LN metastases than for cervical and abdominal LN metastases. This result may be correlated with the worse prognosis of thoracic $\mathrm{LN}$ metastases (Fig. 1d).

There are concerns that surgical complications could reduce long-term survival. It has been shown that the survival rates of patients who developed postoperative complications after curative resection for primary ESCC were significantly poorer than those of patients who did not [28, 29]. To date, no studies of ESCC have addressed the question of whether postoperative complications after resection for recurrent lesions have an impact on long-term survival. During the present study, severe complications after surgical resection for recurrent lesions were correlated with a poor prognosis (Fig. 3b). During the multivariate analysis, the occurrence of severe complications (Clavien-Dindo 
(a) pN stage at primary surgery

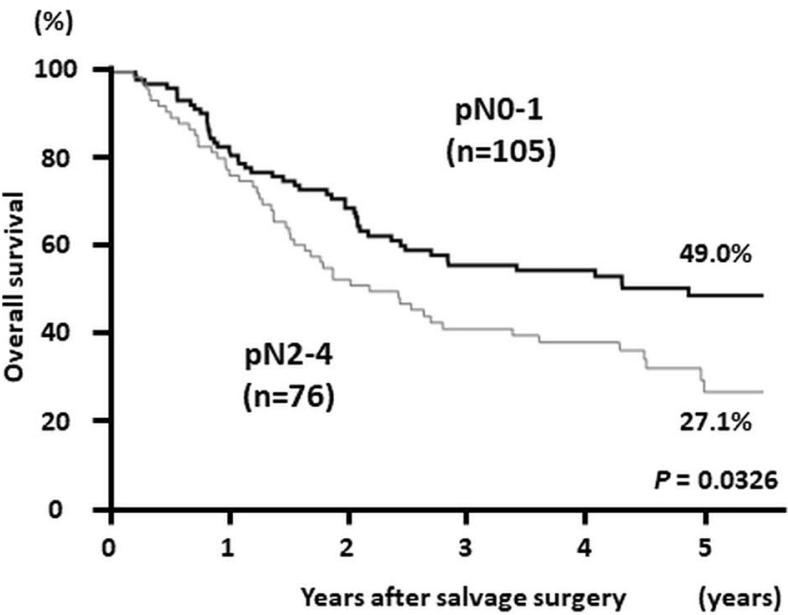

(b) Number of recurrent organ

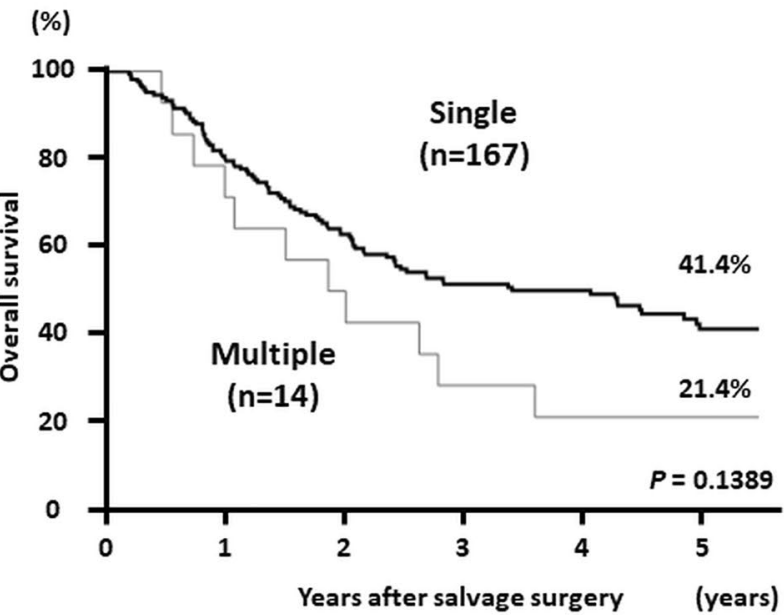

Number at risk

pN0-1 105

pN2-4 76

\begin{tabular}{|c|c|c|}
\hline 84 & 66 & 41 \\
\hline \multirow{2}{*}{\multicolumn{2}{|c|}{58}} & 29 \\
\hline & & MST (days) \\
\hline & pN0-1 & 1767 \\
\hline & pN2-4 & 788 \\
\hline
\end{tabular}

Number at risk

$\begin{array}{lllllll}\text { Single } & 167 & 132 & 98 & 74 & 61 & 35\end{array}$

$\begin{array}{lllllll}\text { Multiple } & 14 & 10 & 6 & 4 & 3 & 2\end{array}$

(c)

Residual tumor

(d)

\begin{tabular}{|c|c|}
\hline & MST (days) \\
\hline Single & 1481 \\
\hline Multiple & 702.5 \\
\hline
\end{tabular}
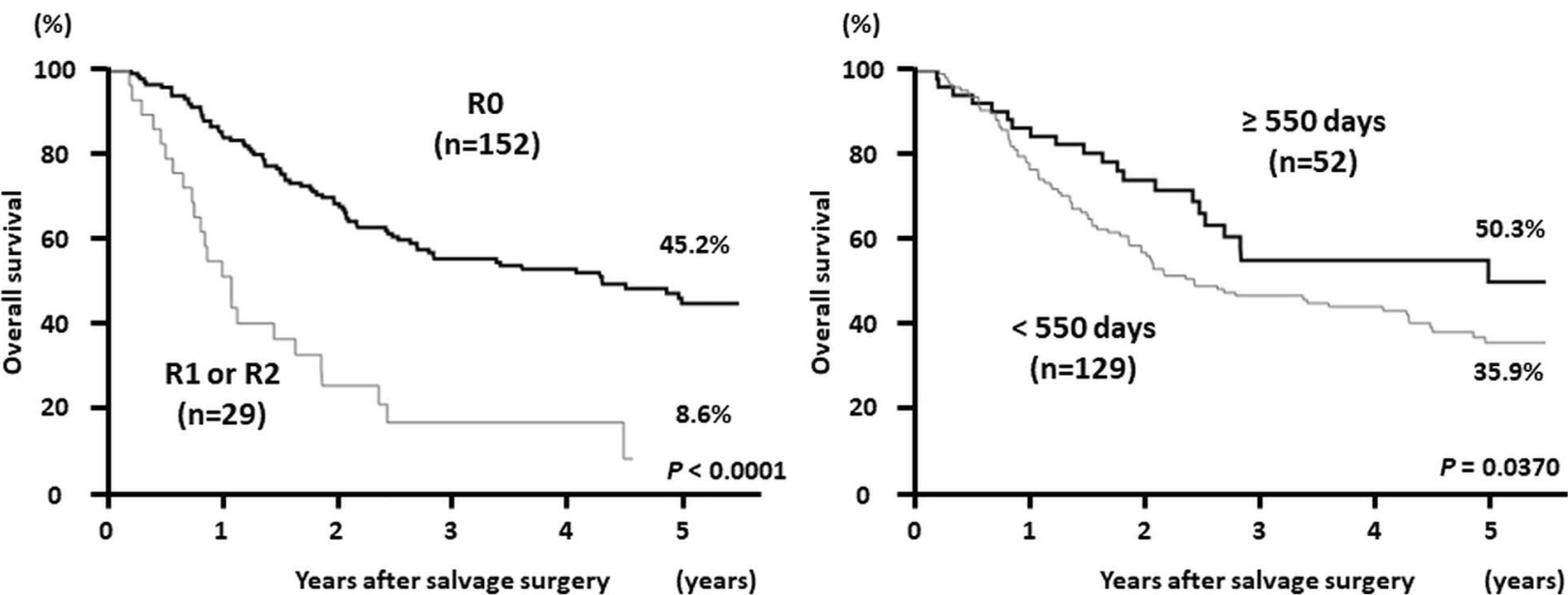

Number at risk

$\begin{array}{lllllllll}\text { R1 or R2 } & 29 & 14 & 7 & 4 & 4 & 0 & <550 & 12\end{array}$

\begin{tabular}{|c|c|}
\hline & MST (days) \\
\hline R0 & 1564 \\
\hline R1 or R2 & 387 \\
\hline
\end{tabular}

$\begin{array}{ccccc}43 & 32 & 20 & 16 & 9 \\ 99 & 72 & 58 & 48 & 28\end{array}$

\begin{tabular}{|c|c|}
\hline & MST (days) \\
\hline$\geq 550$ days & - \\
\hline$<550$ days & 883 \\
\hline
\end{tabular}


4Fig. 2 Kaplan-Meier curves of overall survival after surgical resection for recurrent lesions for a patients with pN0-1 and pN2-4 at primary esophagectomy, b patients who underwent resection and had single-organ metastases and multiple-organ metastases, and $\mathbf{c}$ patients who underwent radical surgery (R0) and non-curative resection (R1 or R2). d Kaplan-Meier curves of overall survival based on the length of the recurrence-free interval after primary esophagectomy. The cut-off value for recurrence-free interval was 550 days, which was obtained by the receiver-operating characteristic curve analysis. $R O$ radical resection, $R 1$ microscopic residual disease, $R 2$ macroscopic residual disease, $M S T$ median survival time

grade $\geq$ IIIa) after resection for recurrent lesions was identified as an independent prognostic factor for poor OS $(P=0.0420)$ (Table 3$)$. These results suggest that excessively invasive surgery should be avoided through appropriate evaluation of the anatomical features of the recurrent site and the general condition of the patient. However, further studies are necessary to confirm the results, because the number of patients in this study with severe complications was too small $(n=7)$.

We also evaluated the efficacy of perioperative treatments such as chemotherapy, radiotherapy, and chemoradiotherapy before or after surgical resection for recurrent lesions. These therapies did not improve the OS after surgical resection for recurrent lesions. On the other hand, OS in the Yes group after surgery for recurrent lesions tended to be inferior to
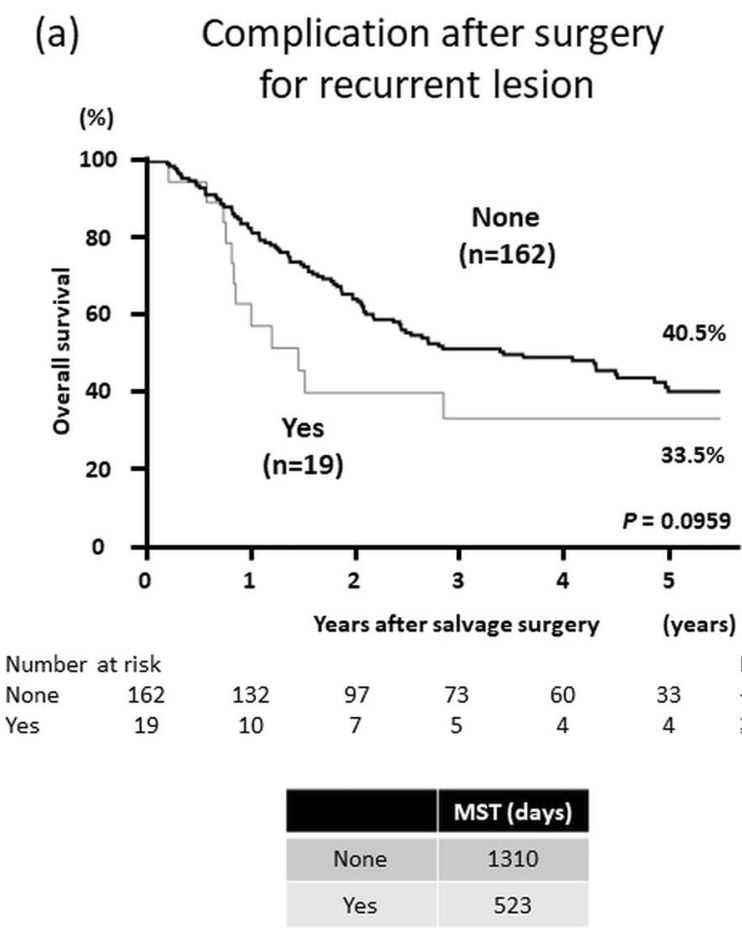

Fig. 3 Kaplan-Meier curves of overall survival after surgical resection for recurrent lesions in patients with and without complications after surgical resection for recurrent lesions. a Patients with and with- that in the None group (Fig. 4b). The rates of R0 resection $(P=0.0069)$ and single-organ recurrence $(P=0.0095)$ were significantly lower in the Yes group than in the None group (Online Resources 5 and 6). The low R0 resection rate may have affected the decision to perform postoperative treatment and the prognosis. Results of subgroup analyses also did not show significant changes in the prognosis, although patients who had multiple recurrent lesions or multipleorgan involvement and those with lung metastasis tended to have a favorable prognosis after undergoing perioperative chemotherapy and/or radiotherapy after recurrence. Previous reports and our study suggest that perioperative treatment might not be necessary when R0 resection is achieved for a single recurrent lesion. However, further studies are required to ascertain whether perioperative chemotherapy and/or radiotherapy should be indicated for patients with multiple recurrent lesions or multiple-organ involvement.

A limitation of this study was that it was retrospective. In addition, the present study did not compare the outcomes of surgical resection for recurrent lesions with the outcomes of other therapies such as chemotherapy, radiotherapy, or chemoradiotherapy. Therefore, we cannot decisively conclude that surgical resection for recurrent lesions is superior to other therapies. However, the overall long-term prognosis for the 186 patients in the present study was more favorable than the generally known data for the prognosis after

\section{(b) Severe complication ( $C D \geq I I I a)$ after surgery for recurrent lesion}$$
\text { (\%) }
$$

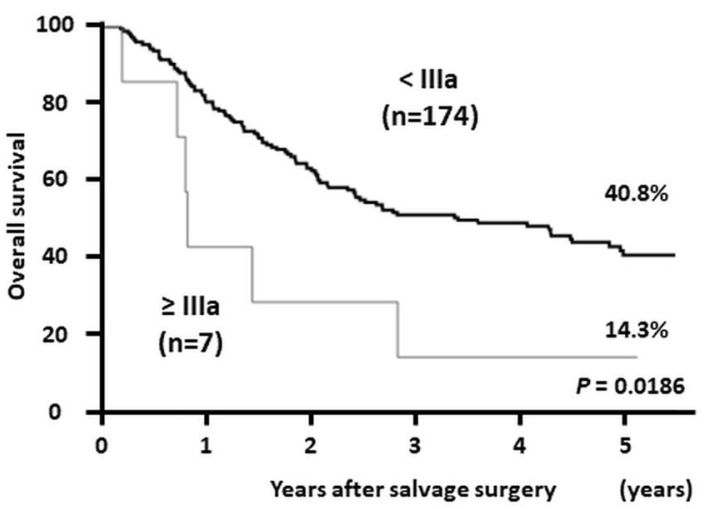

Number at risk

$\begin{array}{lcccccc}<\text { IIla } & 174 & 139 & 102 & 77 & 63 & 36 \\ \geq \text { Illa } & 7 & 3 & 3 & 1 & 1 & 1\end{array}$

out complications and all Clavien-Dindo grades. b Patients with and without severe complications (Clavien-Dindo grade $\geq$ IIIa). MST median survival time, $C D$ Clavien-Dindo grade 


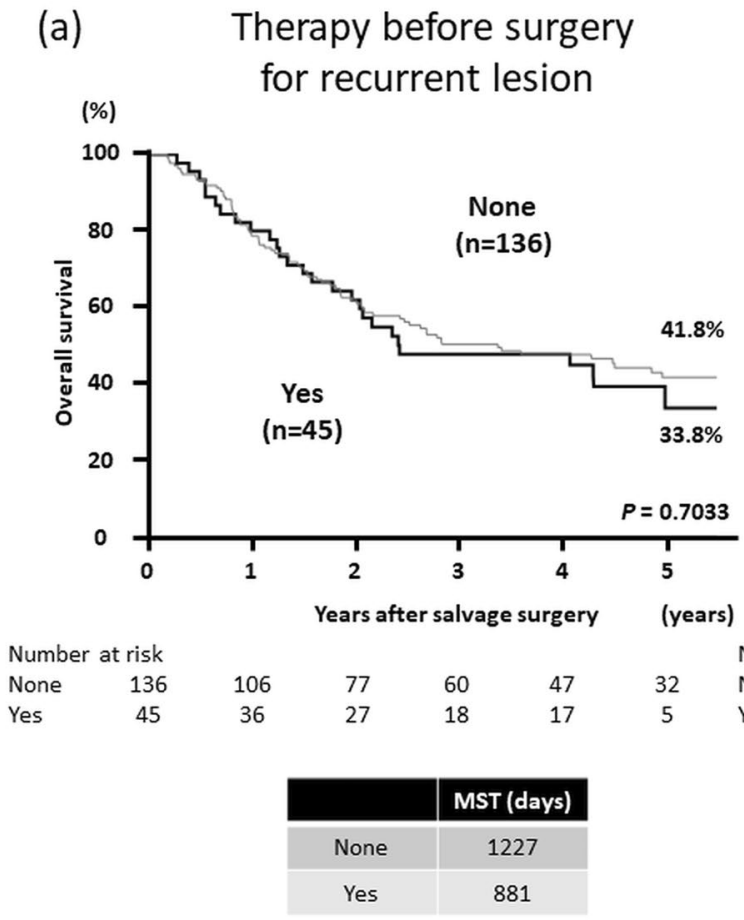

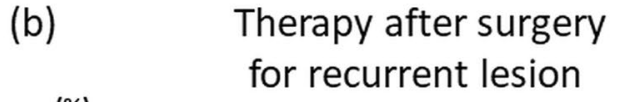

(\%)

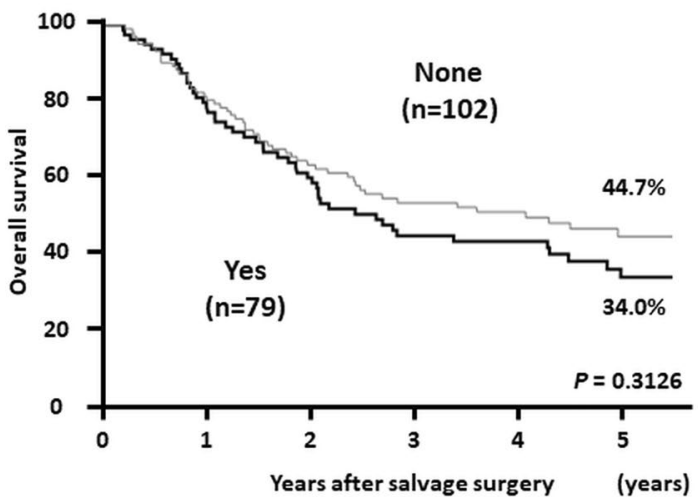

Number at risk

$\begin{array}{lllllll}\text { None } & 102 & 82 & 60 & 47 & 37 & 21\end{array}$
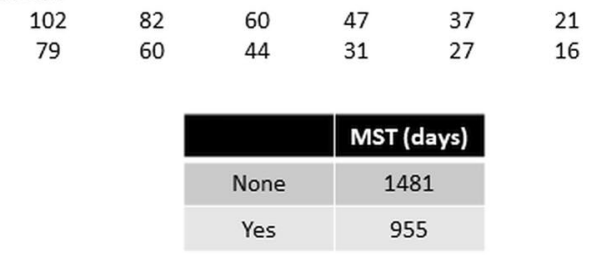

Fig. 4 Kaplan-Meier curves of overall survival after surgical resection for recurrent lesions according to treatment with chemotherapy and/or radiotherapy $\mathbf{a}$ before and $\mathbf{b}$ after surgical resection for recurrent lesions. MST median survival time

Table 3 Univariate and multivariate analyses for overall survival

\begin{tabular}{|c|c|c|c|c|}
\hline \multirow[t]{2}{*}{ Factor } & \multicolumn{2}{|l|}{ Univariate analysis } & \multicolumn{2}{|l|}{ Multivariate analysis } \\
\hline & HR (95\% CI) & $P$ value & $\mathrm{HR}(95 \% \mathrm{CI})$ & $P$ value \\
\hline Female (vs male) & $0.744(0.396-1.284)$ & 0.3027 & $0.789(0.416-1.383)$ & 0.4245 \\
\hline Age $\geq 65$ y (vs $<65$ y) & $0.975(0.659-1.433)$ & 0.8962 & $0.972(0.655-1.437)$ & 0.8889 \\
\hline pT 0-2 (vs pT 3-4) & $0.764(0.517-1.125)$ & 0.1733 & $1.115(0.720-1.725)$ & 0.6249 \\
\hline pN 0-1 (vs pN 2-4) & $0.659(0.448-0.970)$ & 0.0348 & $0.594(0.391-0.902)$ & 0.0146 \\
\hline LN (vs other organs) & $0.840(0.571-1.245)$ & 0.3819 & $0.655(0.405-1.066)$ & 0.0881 \\
\hline Lung (vs other organs) & $0.647(0.386-1.033)$ & 0.0687 & $0.529(0.292-0.932)$ & 0.0274 \\
\hline Solitary lesion (vs multiple) & $0.701(0.453-1.122)$ & 0.1346 & $0.711(0.408-1.332)$ & 0.2723 \\
\hline Single organ (vs multiple) & $0.626(0.350-1.241)$ & 0.1678 & $1.220(0.511-2.983)$ & 0.6543 \\
\hline Recurrence-free interval $\geq 550$ days & $0.604(0.365-0.956)$ & 0.0306 & $0.584(0.348-0.941)$ & 0.0266 \\
\hline $\mathrm{R} 0$ resection (vs R1 or R2) & $0.299(0.190-0.487)$ & $<0.0001$ & $0.389(0.234-0.669)$ & 0.0009 \\
\hline PC $<$ IIIa (surgery for recurrence) & $0.384(0.183-0.987)$ & 0.0472 & $0.352(0.153-0.959)$ & 0.0420 \\
\hline
\end{tabular}

PC Postoperative complication after surgical resection for recurrent lesion (Clavien-Dindo classification) recurrence $[4,5,13]$. The median survival time after surgical resection for recurrent lesions was 1030 days and the 5 -year OS rate was $39.8 \%$ for 181 patients treated at specialized institutes in Japan. Therefore, improved prognoses may be expected after surgical resection for recurrent lesions in patients with the favorable prognostic factors identified during this study. No multi-institutional collaborative study of the resection of recurrent lesions in patients with esophageal carcinoma has included a similar number of cases as this study. Therefore, we believe that the results of the present study will provide meaningful information that can guide decision-making treatment strategies in clinical practice.

In conclusion, the results of our study suggest that surgical resection of recurrent lesions after curative esophagectomy is a useful treatment option, particularly for ESCC patients with lower $\mathrm{pN}$ stage, lung metastasis, long recurrence-free interval, and technically resectable recurrent lesions. When considering resection for recurrent lesions, 
careful attention should be focused on minimizing surgical risks as much as possible.

Supplementary Information The online version contains supplementary material available at https://doi.org/10.1007/s10388-021-00878-2.

Acknowledgements We thank the following 36 institutes that cooperated by providing completed questionnaires: Akita University, Oita University, Osaka General Medical Center, Osaka City University, Okayama University, Kagawa Prefectural Central Hospital, Kanazawa University, Kawasaki Medical School General Medical Center, The Cancer Institute Hospital of Japanese Foundation for Cancer Research, Kansai Rosai Hospital, National Hospital Organization Kyushu Cancer Center, Kitakyushu Municipal Medical Center, Kitano Hospital, The Tazuke Kofukai Medical Research Institute, Kindai University, Kumamoto University, Kobe University, Shizuoka Cancer Center Hospital and Research Institute, Shizuoka General Hospital, Jichi Medical University, Shimane University, Tokai University, Tokyo Medical and Dental University, Medical Hospital, Nagaoka Chuo General Hospital, Nagoya City University, Nagoya University, Nara Medical University, Niigata Cancer Center Hospital, Nippon Medical School, Hiroshima City Asa Hospital, Research Institute for Radiation Biology and Medicine, Hiroshima University, National Defense Medical College, Mie University, Yamagata University Hospital, Yamaguchi University, University of Yamanashi Hospital, and Dokkyo Medical University Hospital. We would like to thank Editage (www.editage. com) for English language editing.

\section{Declarations}

Conflict of interest The authors declare that they have no conflicts of interest and that no funding was received for this study.

Ethical statement All procedures followed were in accordance with the ethical standards of the responsible committee on human experimentation (institutional and national) and with the Helsinki Declaration of 1964 and later versions. Informed consent or substitute for it was obtained from all patients for being included in the study.

Open Access This article is licensed under a Creative Commons Attribution 4.0 International License, which permits use, sharing, adaptation, distribution and reproduction in any medium or format, as long as you give appropriate credit to the original author(s) and the source, provide a link to the Creative Commons licence, and indicate if changes were made. The images or other third party material in this article are included in the article's Creative Commons licence, unless indicated otherwise in a credit line to the material. If material is not included in the article's Creative Commons licence and your intended use is not permitted by statutory regulation or exceeds the permitted use, you will need to obtain permission directly from the copyright holder. To view a copy of this licence, visit http://creativecommons.org/licenses/by/4.0/.

\section{References}

1. Miyata H, Yamasaki M, Kurokawa Y, et al. Survival factors in patients with recurrence after curative resection of esophageal squamous cell carcinomas. Ann Surg Oncol. 2011;18:3353-61.

2. Abate E, DeMeester SR, Zehetner J, et al. Recurrence after esophagectomy for adenocarcinoma: defining optimal follow-up intervals and testing. J Am Coll Surg. 2010;210:428-35.
3. Kato H, Fukuchi M, Miyazaki T, et al. Classification of recurrent esophageal cancer after radical esophagectomy with two- or threefield lymphadenectomy. Anticancer Res. 2005;25:3461-7.

4. Mariette C, Balon JM, Piessen G, et al. Pattern of recurrence following complete resection of esophageal carcinoma and factors predictive of recurrent disease. Cancer. 2003;97:1616-23.

5. Kato F, Monma S, Koyanagi K, et al. Long-term outcome after resection for recurrent oesophageal cancer. J Thorac Dis. 2018;10:2691-9.

6. Chen J, Yin W, Yao H, et al. Salvage treatment for lymph node recurrence after radical resection of esophageal squamous cell carcinoma. Radiat Oncol. 2019;14:169.

7. Watanabe M, Nishida K, Kimura Y, et al. Salvage lymphadenectomy for cervical lymph node recurrence after esophagectomy for squamous cell carcinoma of the thoracic esophagus. Dis Esophagus. 2012;25:62-6.

8. Nakamura T, Ota M, Narumiya K, et al. Multimodal treatment for lymph node recurrence of esophageal carcinoma after curative resection. Ann Surg Oncol. 2008;15:2451-7.

9. Wang Z, Lin S, Wang F, et al. Salvage lymphadenectomy for isolated cervical lymph node recurrence after curative resection of thoracic esophageal squamous cell carcinoma. Ann Transl Med. 2019;7:238

10. Ma X, Zhao K, Guo W, et al. Salvage lymphadenectomy versus salvage radiotherapy/chemoradiotherapy for recurrence in cervical lymph node after curative resection of esophageal squamous cell carcinoma. Ann Surg Oncol. 2015;22:624-9.

11. Shimada A, Tsushima T, Tsubosa Y, et al. Validity of surgical resection for lymph node or pulmonary recurrence of esophageal cancer after definitive treatment. World J Surg. 2019;43:1286-93.

12. Kosuga T, Shiozaki A, Fujiwara $\mathrm{H}$, et al. Treatment outcome and prognosis of patients with lymph node recurrence of thoracic esophageal squamous cell carcinoma after curative resection. World J Surg. 2011;35:798-804.

13. Su XD, Zhang DK, Zhang X, et al. Prognostic factors in patients with recurrence after complete resection of esophageal squamous cell carcinoma. J Thorac Dis. 2014;6:949-57.

14. Nakagawa $S$, Kanda $T$, Kosugi $S$, et al. Recurrence pattern of squamous cell carcinoma of the thoracic esophagus after extended radical esophagectomy with three-field lymphadenectomy. J Am Coll Surg. 2004;198:205-11.

15. Hsu PK, Wang BY, Huang CS, et al. Prognostic factors for postrecurrence survival in esophageal squamous cell carcinoma patients with recurrence after resection. J Gastrointest Surg. 2011;15:558-65.

16. Koizumi W, Kitago M, Shinoda M, et al. Successful resection of pancreatic metastasis from oesophageal squamous cell carcinoma: a case report and review of the literature. BMC Cancer. 2019;19:320.

17. Hiyoshi Y, Morita M, Kawano H, et al. Clinical significance of surgical resection for the recurrence of esophageal cancer after radical esophagectomy. Ann Surg Oncol. 2015;22:240-6.

18. Ichida $\mathrm{H}$, Imamura $\mathrm{H}$, Yoshimoto J, et al. Pattern of postoperative recurrence and hepatic and/or pulmonary resection for liver and/ or lung metastases from esophageal carcinoma. World J Surg. 2013;37:398-407.

19. Dindo D, Demartines N, Clavien PA. Classification of surgical complications: a new proposal with evaluation in a cohort of 6336 patients and results of a survey. Ann Surg. 2004;240:205-13.

20. Clavien PA, Barkun J, de Oliveira ML, et al. The Clavien-Dindo classification of surgical complications: five-year experience. Ann Surg. 2009;250:187-96.

21. Shioyama Y, Nakamura K, Ohga S, et al. Radiation therapy for recurrent esophageal cancer after surgery: clinical results and prognostic factors. Jpn J Clin Oncol. 2007;37:918-23. 
22. Jingu K, Nemoto K, Matsushita H, et al. Results of radiation therapy combined with nedaplatin (cis-diammine-glycoplatinum) and 5-fluorouracil for postoperative locoregional recurrent esophageal cancer. BMC Cancer. 2006;6:50.

23. Ni W, Yang J, Deng W, et al. Patterns of recurrence after surgery and efficacy of salvage therapy after recurrence in patients with thoracic esophageal squamous cell carcinoma. BMC Cancer. 2020;20:144.

24. Zhu YL, Li Q, Gao JM, et al. A retrospective evaluation of radiotherapy for the treatment of local esophageal squamous cell carcinoma recurrence after initial complete surgical resection. J Investig Med. 2013;61:34-9.

25. Yano M, Takachi K, Doki Y, et al. Prognosis of patients who develop cervical lymph node recurrence following curative resection for thoracic esophageal cancer. Dis Esophagus. 2006;19:73-7.

26. Takemura M, Kaibe N, Takii M, et al. Treatment outcomes of patients with cervical lymph node recurrence after esophagectomy for esophageal cancer. Gan To Kagaku Ryoho. 2014;41:2010-2.
27. Komatsu S, Shioaki Y, Ichikawa D, et al. Survival and clinical evaluation of salvage operation for cervical lymph node recurrence in esophageal cancer. Hepatogastroenterology. 2005;52:796-9.

28. Saeki H, Tsutsumi S, Tajiri H, et al. Prognostic significance of postoperative complications after curative resection for patients with esophageal squamous cell carcinoma. Ann Surg. 2017;265:527-33.

29. Baba Y, Yoshida N, Shigaki H, et al. Prognostic impact of postoperative complications in 502 patients with surgically resected esophageal squamous cell carcinoma: a retrospective single-institution study. Ann Surg. 2016;264:305-11.

Publisher's Note Springer Nature remains neutral with regard to jurisdictional claims in published maps and institutional affiliations. 